Małgorzata Dybowska, Monika Szturmowicz, Paweł Kuca, Barbara Kazanecka, Janusz Burakowski, Cezary Czajka, Franciszek Grzegorczyk, Renata Langfort, Barbara Burakowska, Piotr Rudziński, Witold Tomkowski

National Tuberculosis and Lung Diseases Research Institute, Warsaw, Poland

\title{
Intrapericardial cisplatin combined with oral colchicine resulting in long term control of malignant pericardial effusion in the course of metastatic renal cancer
}

\author{
The authors declare no finacial disclosure
}

\begin{abstract}
Introduction: Neoplastic pericardial effusion (NPE) represents a common cause of morbidity and mortality in patients with cancer. NPE presents frequently as cardiac tamponade, requiring urgent pericardiocentesis or pericardiotomy, with subsequent pericardial fluid drainage. Despite high effectiveness of such procedures, the recurrence of effusion is noted in $30-60 \%$ of patients. Intrapericardial therapy with cisplatin was found to be effective in NPE due to lung and breast cancer. Its role in cardiac tamponade due to renal cancer is unknown.

Case presentation: We presented an 82-year-old man with renal cancer who was admitted to the Intensive Care Unit because of threatening pericardial tamponade due to NPE.

Urgent subxiphoid pericardiotomy was performed with subsequent evacuation of $1000 \mathrm{ml}$ of bloody fluid. On the inner surface of the pericardium, several pink nodules were found. Histological examination revealed carcinoma clarocellulare. In view of the persistent high drainage of the pericardium, intrapericardial cisplatin therapy was performed. On the first day after surgery, colchicine $0.5 \mathrm{mg} /$ day/po was also introduced. No side effects of the treatment were observed. The patient died 12 months later due to cancer progression and cachexia. No recurrence of pericardial effusion was observed.

Conclusion: This is the first case study demonstrating long-term efficacy and safety of intrapericardial cisplatin combined with oral colchicine in NPE due to metastatic renal cell carcinoma.
\end{abstract}

Key words: neoplastic pericardial effusion, renal cancer, cisplatin, intrapericardial treatment, colchicine

Adv Respir Med. 2018; 86: 197-201

\section{Introduction}

Neoplastic pericardial effusion (NPE) represents a common cause of morbidity and mortality in cancer patients. Over $60 \%$ of NPE is diagnosed in the course of lung cancer, breast cancer and hematologic malignancies [1-3]. NPE presents frequently as cardiac tamponade, requiring urgent pericardiocentesis or pericardiotomy, with subsequent pericardial fluid (PF) drainage [4, 5]. Despite high effectiveness of such procedures, the recurrence of effusion was noted in $30-60 \%$ of patients [6, 7].
Intrapericardial cisplatin therapy was found to be an effective method of prevention of NPE recurrence in lung cancer patients $[8,9]$. The efficacy of such treatment in other solid tumors is poorly documented.

Colchicine, an anti-inflammatory drug, inhibits neutrophils motility and activity at the site of inflammation, decreases the production of IL-1 by monocytes and reduces the release of lysozymes by phagocytes [10]. It is currently recommended as first-line therapy of acute and recurrent benign pericarditis [4]. The COPPS study proved colchi- 
cine efficacy in prevention of post-pericardiotomy syndrome [11]. The role of colchicine in NPE has not been established yet.

The present case report concerns successful intra-pericardial cisplatin treatment combined with oral colchicine therapy in the patient with NPE due to metastatic renal cancer.

\section{Case presentation}

An 82-year-old man, treated for arterial hypertension and hypothyroidism, with a history of right-sided nephrectomy in 2003 due to clear-cell renal carcinoma, with metastatic lung disease treated with sunitinib (2009-2012) and everolimus (2012-2013) was admitted to the Intensive Care Unit (ICU) in June 2013 because of threatening cardiac tamponade. Two weeks earlier the patient had noticed a decrease in exercise tolerance, accompanied by a dry cough.

On admission to ICU the man was in poor general condition, with marked weight loss about $5 \mathrm{~kg}$ in 3 months (BMI 18). Physical examination revealed blood pressure 150/80, heart rate 90/min. On auscultation - dull heart sounds, and wheezing over the lung fields were heard. Liver was enlarged, palpable $4 \mathrm{~cm}$ below the right costal margin. Ortopnea and hemodynamic instability were not found.

Laboratory analysis revealed anemia (E 3.85 $\times 10^{12} / \mathrm{l}$, hemoglobin $9.4 \mathrm{~g} \%$ ), elevated levels of D-dimer (2674 ug/l), increased GGTP (95 U/l) and
NT-proBNP (541 pg/ml). TSH was $2.6 \mathrm{mIU} / \mathrm{ml}$, fT4 - $17.8 \mathrm{pmol} / \mathrm{l}$ (within normal limits).

Chest X-ray showed significantly enlarged cardiac silhouette, bilateral pleural effusion, and bilateral lung infiltrative lesions, suggesting metastatic lung disease (Fig. 1).

ECG revealed sinus rhythm of about $85 / \mathrm{min}$., left anterior hemi block, right branch bundle block. Low QRS voltage as well as signs of electrical alternans were also recorded (Fig. 2).

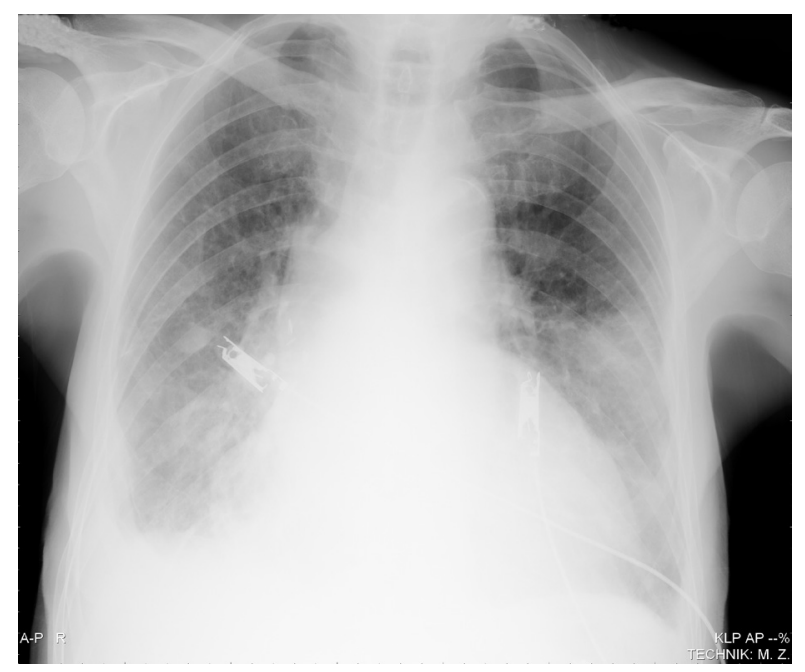

Figure 1. Chest X-ray: significantly enlarged cardiac silhouette, bilateral pleural effusion, and bilateral lung infiltrative lesions, suggesting metastatic lung disease

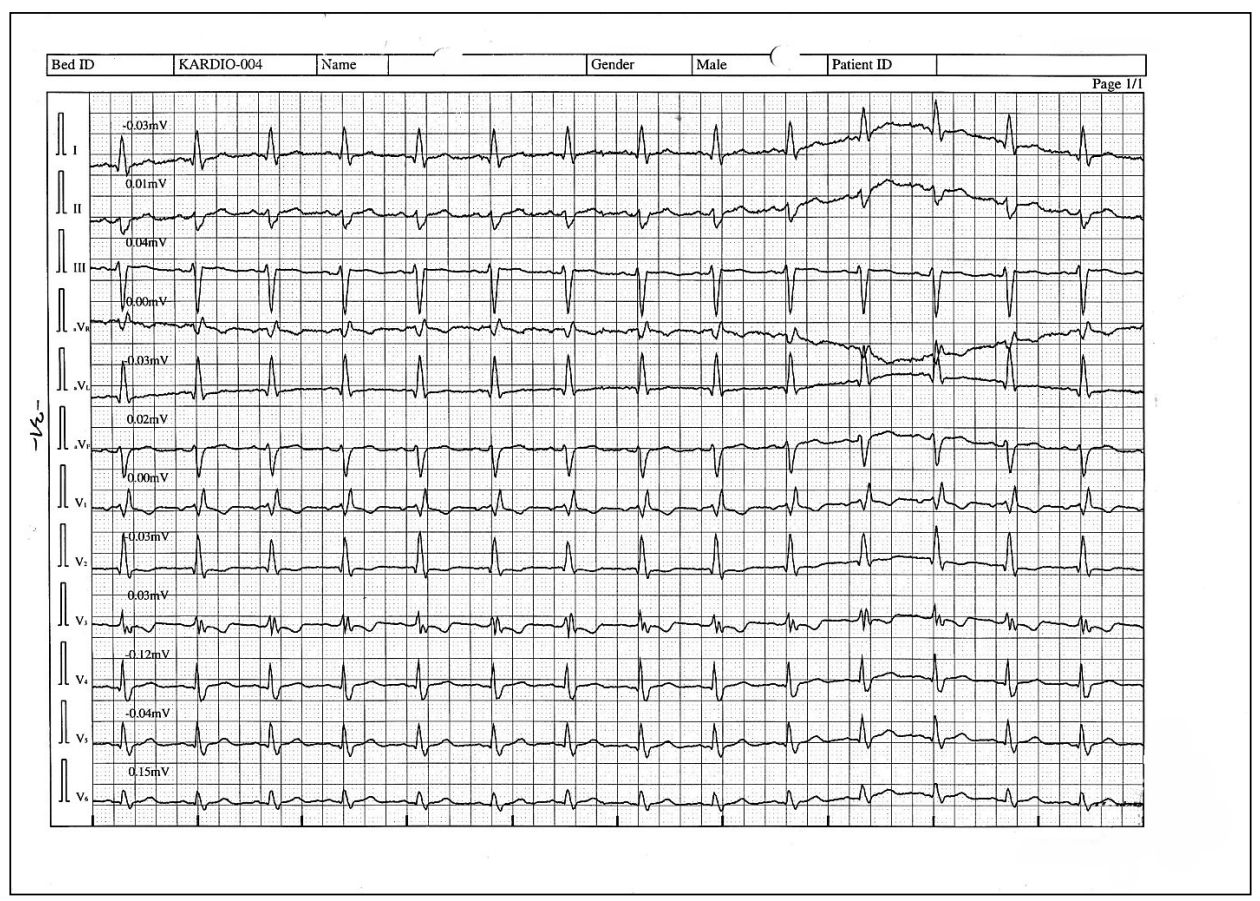

Figure 2. ECG Sinus rhythm about 85 per minute. Left axis pathological. Left anterior hemiblock. Right bundle branch block. Electrical alternans 


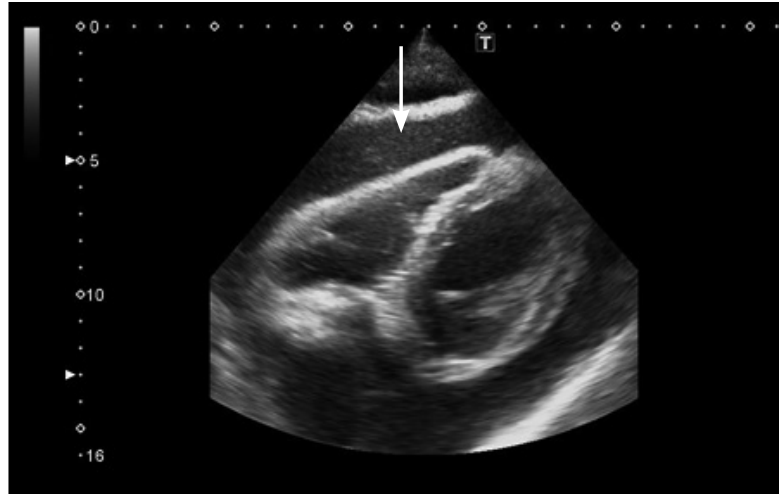

Figure 3. Echocardiography pre- treatment: fluid around the whole heart (arrow)

Bedside echocardiography demonstrated the presence of pericardial fluid (a layer of 9-16 $\mathrm{mm}$ ), with elements of fibrin, surrounding the whole heart. Moreover, right atrium diastolic collapse was noted (Fig. 3).

Threatening cardiac tamponade was diagnosed. As the amount of fluid was not very large and the widest layer was found over the right ventricle, the patient was proposed subxiphoid pericardiotomy, but he was initially reluctant.

On the second day he experienced an episode of atrial fibrillation with a decrease in blood pressure to $90 / 60 \mathrm{~mm} \mathrm{Hg}$. Urgent subxiphoid pericardiotomy was performed, $1000 \mathrm{ml}$ of bloody fluid was removed. Several pink nodules were found on the inner surface of pericardium. Histological examination of pericardial specimen revealed multiple carcinoma cell clusters - $\mathrm{Ca}$ clarocellulare. Pericardial fluid cytological examination was positive. Pericardial fluid cultures were negative. Thus NPE was confirmed.

Due to persistent high pericardial drainage in subsequent days (250-450 ml of bloody fluid/ /day), intrapericardial cisplatin therapy was commenced. The drug was given on 5 consecutive days. Each dose consisted of $10 \mathrm{mg}$ of cisplatin dissolved in $20 \mathrm{ml}$ of $0.9 \% \mathrm{NaCl}$ administered by a catheter into the pericardial sac. The drain was clamped for 24 hours, then the fluid was removed and the dose of cisplatin was repeated. Starting from the first day after surgery, colchicine $0.5 \mathrm{mg} /$ day was introduced. No complications of treatment were observed.

Control echocardiographic examination confirmed significant resolution of PF. No signs of pericardial constriction were found (Fig. 4). Control chest X-ray examination disclosed the decrease of cardiac dimensions and partial regression of pleural fluid (Fig. 5).

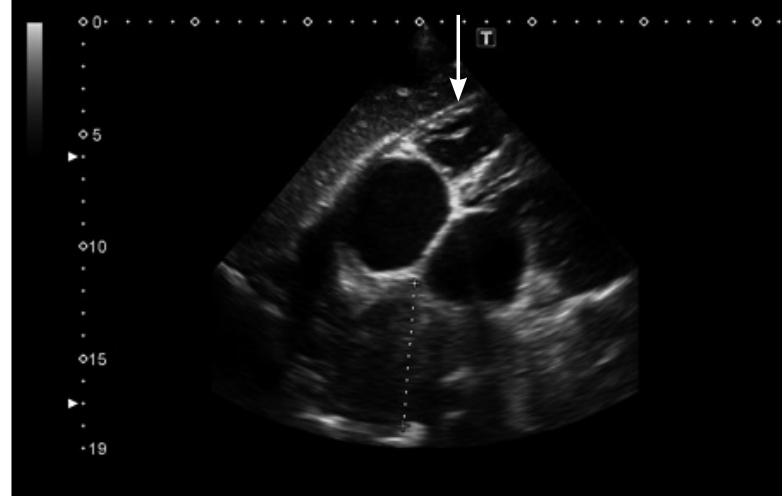

Figure 4. Echocardiography post- treatment: no fluid around the pericardium (arrow)

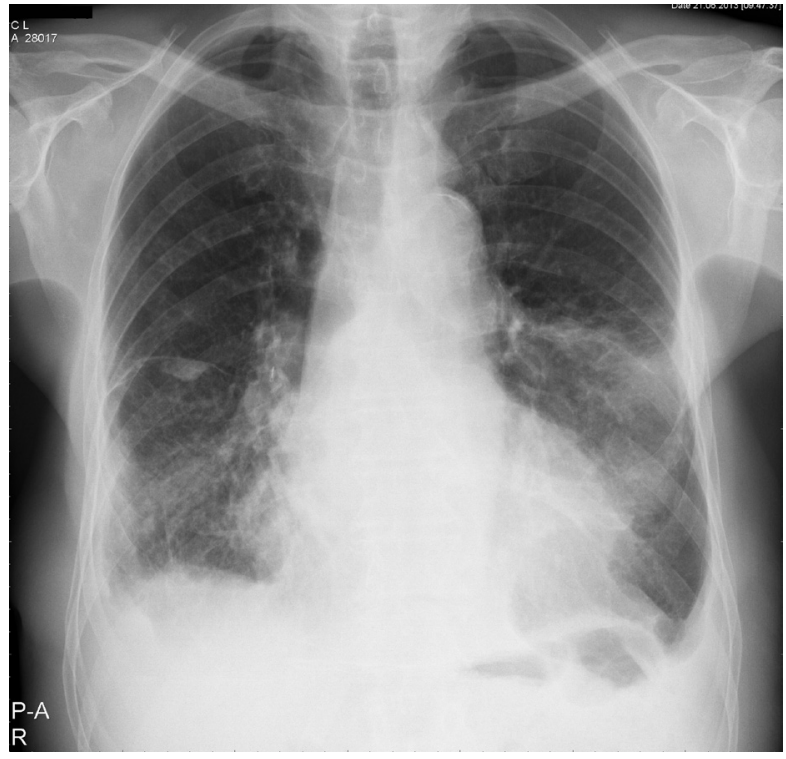

Figure 5. Control chest X-ray examination revealed the decrease of cardiac dimensions and partial regression of pleural fluid

On the $13^{\text {th }}$ postoperative day pericardial drain was removed. The patient was discharged, and he continued the treatment with colchicine. Despite gradual progression of renal cell carcinoma, pericardial disease has been well controlled until death. Last echocardiographic examination revealed the presence of additional echoes in the left atrium and left ventricle, representing probably intracardiac metastatic lesions. The patient survived 12 months from intrapericardial cisplatin therapy.

\section{Discussion}

The ideal treatment for NPE ensures the complete removal of fluid, relief of symptoms, prevention of recurrent effusion, influence on the local neoplastic disease and survival benefit [12]. 
Pericardiotomy with PF drainage was not a sufficient method of therapy in the presented patient due to subsequent large daily production of PF.

Improvement of local disease control and prevention of PF recurrence may be achieved by intrapericardial instillation of cytotoxic agents such as cisplatin, thiotepa, bleomycin $[8,9$, 13-16]. Recently, intrapericardial activity of anti-angiogenic factor - bevacizumab was reported in NPE [17].

The rationale for intrapericardial instillation of antineoplastic drug is to provide its higher concentration than those achieved in the course of systemic therapy. So far no randomized controlled trials evaluating the efficacy and safety of intrapericardial chemotherapy have been performed, and that is why all recommendations are based only on the results of small observational studies.

Intrapericardial treatment tailored to the type of the tumor indicate that administration of cisplatin has been most efficient in NPE due to lung cancer $[8,9,13]$, and intrapericardial instillation of thiotepa has been more effective in NPE due to breast cancer $[18,19]$.

There is no evidence for the efficacy of systemic chemotherapy with cisplatin in patients with renal cell carcinoma, and the experience with intrapericardial therapy in this type of tumor is lacking. Nevertheless, we decided to apply intrapericardial cisplatin, having in mind its good therapeutic and safety profile in other solid tumors.

Colchicine was introduced as an early add -on therapy to improve local anti-inflammatory effect. There is not much data concerning colchicine therapy in NPE. Ng et al. [20] reported successful colchicine use in NPE that developed in the course of malignant myeloma [20]. In this patient, pericardiocentesis, followed by surgical approach with the creation of pericardial window, were ineffective. Colchicine at a dose of $1 \mathrm{mg} /$ day, stopped pericardial drainage after 5 days of treatment.

In our patient intrapericardial cisplatin administration and colchicine therapy resulted in gradual, almost complete resolution of pericardial fluid. Moreover, prolonged colchicine therapy successfully prevented effusion recurrence for the next 12 months.

No side effects of colchicine treatment were observed in our patient. Prolonged colchicine therapy has been well tolerated in patients with other diseases such as familial Mediterranean fever or in recurrent benign pericarditis [21, 22]. Never- theless, it is important to adjust the daily dose of the drug to the patient's age and weight [4]. The dose of colchicine was reduced in our patient to $0.5 \mathrm{mg} / \mathrm{d}$, due to low weight and advanced age.

Drug interactions have to be taken into consideration in the course of prolonged colchicine use, especially with macrolides and statins [4]. The interaction between TKI-inhibitors and colchicines has been also reported, thus lowering its dose to $0.3 \mathrm{mg} /$ day was proposed [23].

\section{Summary}

Intrapericardial cisplatin therapy combined with oral colchicine demonstrated an excellent and prolonged local anti-inflammatory effect in cardiac tamponade due to metastatic renal cell carcinoma.

\section{Conflict of interest}

The authors declare no financial disclosure.

\section{References:}

1. Maisch B, Seferović PM, Ristić AD, et al. Task Force on the Diagnosis and Management of Pricardial Diseases of the European Society of Cardiology. Guidelines on the diagnosis and management of pericardial diseases executive summary; The Task force on the diagnosis and management of pericardial diseases of the European society of cardiology. Eur Heart J. 2004; 25(7): 587-610, doi: 10.1016/j.ehj.2004.02.002, indexed in Pubmed: 15120056.

2. Pawlak-Cieślik A, Szturmowicz M, Fijałkowska A, et al. Diagnosis of malignant pericarditis: a single centre experience. Kardiol Pol. 2012; 70(11): 1147-1153, indexed in Pubmed: 23180523.

3. Çelik S, Lestuzzi C, Cervesato E, et al. Systemic chemotherapy in combination with pericardial window has better outcomes in malignant pericardial effusions. J Thorac Cardiovasc Surg. 2014; 148(5): 2288-2293, doi: 10.1016/j.jtcvs.2014.04.031, indexed in Pubmed: 24836991.

4. Charron P, Adler Y, Imazio M, et al. Società Europea di Cardiologia, ESC Scientific Document Group . 2015 ESC Guidelines for the diagnosis and management of pericardial diseases: The Task Force for the Diagnosis and Management of Pericardial Diseases of the European Society of Cardiology (ESC)Endorsed by: The European Association for Cardio-Thoracic Surgery (EACTS). Eur Heart J. 2015; 36(42): 2921-2964, doi: 10.1093/ eurheartj/ehv318, indexed in Pubmed: 26320112.

5. Ristić AD, Imazio M, Adler Y, et al. Triage strategy for urgent management of cardiac tamponade: a position statement of the European Society of Cardiology Working Group on Myocardial and Pericardial Diseases. Eur Heart J. 2014; 35(34): 2279-2284, doi: 10.1093/eurheartj/ehu217, indexed in Pubmed: 25002749.

6. Sánchez-Enrique C, Nuñez-Gil IJ, Viana-Tejedor A, et al. Cause and long-term outcome of cardiac tamponade. Am J Cardiol. 2016; 117(4): 664-669, doi: 10.1016/j.amjcard.2015.11.023, indexed in Pubmed: 26718232.

7. Virk SA, Chandrakumar D, Villanueva C, et al. Systematic review of percutaneous interventions for malignant pericardial effusion. Heart. 2015; 101(20): 1619-1626, doi: 10.1136/heartjnl-2015-307907, indexed in Pubmed: 26180077.

8. Tomkowski WZ, Wiśniewska J, Szturmowicz M, et al. Evaluation of intrapericardial cisplatin administration in cases with recurrent malignant pericardial effusion and cardiac tamponade. Support Care Cancer. 2004; 12(1): 53-57, doi: 10.1007/ s00520-003-0533-x, indexed in Pubmed: 14505155. 
9. Lestuzzi C, Bearz A, Lafaras C, et al. Neoplastic pericardial disease in lung cancer: impact on outcomes of different treatment strategies. A multicenter study. Lung Cancer. 2011; 72(3): 340-347, doi: 10.1016/j.lungcan.2010.10.013, indexed in Pubmed: 21122938.

10. Ben-Chetrit E, Levy M. Colchicine: 1998 update. Semin Arthritis Rheum. 1998; 28(1): 48-59, indexed in Pubmed: 9726336.

11. Imazio $\mathrm{M}$, Trinchero $\mathrm{R}$, Brucato $\mathrm{A}$, et al. COPPS Investigators. COlchicine for the Prevention of the Post-pericardiotomy Syndrome (COPPS): a multicentre, randomized, double-blind, placebo-controlled trial. Eur Heart J. 2010; 31(22): 2749-2754, doi: 10.1093/eurhearti/ehq319, indexed in Pubmed: 20805112.

12. Patel N, Rafique AM, Eshaghian S, et al. Retrospective comparison of outcomes, diagnostic value, and complications of percutaneous prolonged drainage versus surgical pericardiotomy of pericardial effusion associated with malignancy. Am J Cardiol. 2013; 112(8): 1235-1239, doi: 10.1016/j.amjcard.2013.05.066, indexed in Pubmed: 23827405.

13. Maisch B, Rupp H, Ristic A, et al. Pericardioscopy and epiand pericardial biopsy - a new window to the heart improving etiological diagnoses and permitting targeted intrapericardial therapy. Heart Fail Rev. 2013; 18(3): 317-328, doi: 10.1007/ s10741-013-9382-y, indexed in Pubmed: 23479317.

14. Numico G, Cristofano A, Occelli M, et al. Prolonged drainage and intrapericardial bleomycin administration for cardiac tamponade secondary to cancer-related pericardial effusion. Medicine (Baltimore). 2016; 95(15): e3273, doi: 10.1097/ MD.0000000000003273, indexed in Pubmed: 27082564.

15. Maruyama R, Yokoyama H, Seto T, et al. Catheter drainage followed by the instillation of bleomycin to manage malignant pericardial effusion in non-small cell lung cancer: a multi-institutional phase II trial. J Thorac Oncol. 2007; 2(1): 65-68, doi: 10.1097/JTO.0b013e31802c8260, indexed in Pubmed: 17410012.

16. Kunitoh H, Tamura T, Shibata T, et al. JCOG Lung Cancer Study Group, Tokyo, Japan. A randomised trial of intrapericardial bleomycin for malignant pericardial effusion with lung cancer (JCOG9811). Br J Cancer. 2009; 100(3): 464-469, doi: 10.1038/ sj.bjc.6604866, indexed in Pubmed: 19156149.

17. Chen D, Zhang Y, Shi F, et al. Intrapericardial bevacizumab safely and effectively treats malignant pericardial effusion in advanced cancer patients. Oncotarget. 2016; 7(32): 5243652441, doi: 10.18632/oncotarget.9420, indexed in Pubmed: 27203219.

18. Bishiniotis TS, Antoniadou S, Katseas G, et al. Malignant cardiac tamponade in women with breast cancer treated by pericardiocentesis and intrapericardial administration of triethylenethiophosphoramide (thiotepa). Am J Cardiol. 2000; 86(3): 362-364, indexed in Pubmed: 10922456.

19. Colleoni M, Martinelli G, Beretta F, et al. Intracavitary chemotherapy with thiotepa in malignant pericardial effusions: an active and well-tolerated regimen. J Clin Oncol. 1998; 16(7): 2371-2376, doi: 10.1200/JCO.1998.16.7.2371, indexed in Pubmed: 9667253.

20. Ng T, Gatt A, Pagliuca A, et al. Colchicine: an effective treatment for refractory malignant pericardial effusion. Acta Haematol. 2000; 104(4): 217-219, doi: 10.1159/000046519, indexed in Pubmed: 11279315.

21. Imazio M, Brucato A, Cemin R. A randomized trial of colchicine for acute pericarditis. N Engl J Med. 2013; 369: 1522-1528, indexed in Pubmed: 20934560.

22. Imazio M, Belli R, Brucato A, et al. Efficacy and safety of colchicine for treatment of multiple recurrences of pericarditis (CORP-2): a multicentre, double-blind, placebo-controlled, randomised trial. Lancet. 2014; 383(9936): 2232-2237, doi: 10.1016/S0140-6736(13)62709-9, indexed in Pubmed: 24694983.

23. Abodunde OA, LevakaVeera RR, Desai R, et al. Colchicine toxicity precipitated by interaction with sunitinib. J Clin Pharm Ther. 2013; 38(3): 243-245, doi: 10.1111/jcpt.12047, indexed in Pubmed: 23448320. 